\title{
A Prospective Study to Compare the Maternal and Fetal Outcomes among Sickle Cell Disease and Trait Women
}

\author{
Santosh Kumar Dora ${ }^{1}$, Atal B Dandapat ${ }^{2}$, Benudhar Pande ${ }^{3}$, Ganeshram Bhoi ${ }^{4}$, Bhawana Tiwari ${ }^{5}$
}

\begin{abstract}
Introduction: We conducted a prospective trial to compare the maternal and fetal outcomes between the sickle cell disease (SCD) and sickle cell trait (SCT) pregnant patients.

Materials and methods: From December 2015 to December 2016, a total of 59 patients were diagnosed with SCD and 119 patients with SCT. All the fetal and maternal parameters were compared between them.

Results: A total of 17 (28.8\%) SCD and 5 (4.2\%) SCT patients presented with painful crisis. Acute chest syndrome developed in 9 (15.3\%) of SCD and $1(0.8 \%)$ of SCT cases. Hemolytic crisis was seen in $4(6.8 \%)$ of SCD patients. The incidence of hypertension, preeclampsia, jaundice, blood transfusion during pregnancy, and IUGR among the SCD and SCT patients were $11(18.6 \%)$ vs $5(4.2 \%)(p=0.000), 19(32.2 \%)$ vs $7(5.9 \%)$ $(p=0.000), 15(25.4 \%)$ vs $0(0 \%)(p=0.000), 36(61 \%)$ vs $8(6.7 \%)(p=0.000)$, and $33(55.9 \%)$ vs $21(17.6 \%)(p=0.000)$, respectively. The mean periods of gestation of delivery were significantly lower, i.e., $36.5 \pm 2.76$ weeks for SCD patients compared to $38.2 \pm 2.1$ weeks for SCT patients $(p=0.000)$. Ten $(16.9 \%)$ of SCD and one $(0.8 \%)$ of SCT patients had intrauterine death. Average birth weights of babies delivered were $2142 \pm$ $557.45 \mathrm{~g}$ and $2684 \pm 551.23 \mathrm{~g}$ for SCD and SCT patients, respectively.

Conclusion: Sickle cell anemia causes an increased risk to both mother and fetus. Sickle cell disease women are more prone to develop sickle cell crisis as well as increased obstetrical complication. A preconceptional counseling of all sickling women with a multidisciplinary approach can prevent many of the maternal and fetal complications during pregnancy.

Keywords: Sickle cell anemia, Sickle cell crisis, Sickle cell disease, Sickle cell trait.

Journal of South Asian Federation of Obstetrics and Gynaecology (2019): 10.5005/jp-journals-10006-1724
\end{abstract}

\section{INTRODUCTION}

Sickle cell disease (SCD) is an autosomal recessive disorder leading to the structural defect in the hemoglobin. It's most prevalent in the sub-Saharan Africa, Middle East, and parts of India. ${ }^{1}$ But nowadays because of increased connectivity and migration of people, it has become a disease of global importance. ${ }^{2}$ Sickle cell disease comprises of a group of diseases, which includes homozygous HbSS, combination of $\mathrm{HbS}$ with a normal $\mathrm{HbA}$ (sickle cell trait), or different heterozygous combinations of $\mathrm{HbS}$ with either $\beta$ thalassemia, $\mathrm{HbC}$, $\mathrm{HBd}, \mathrm{HBe}$, and O Arab. Globally, more than 300,000 babies were born from the mother caring the mutated gene. ${ }^{3}$ Patients with either homozygous SCD or compound heterozygous characterized with chronic hemolysis, vaso occlusive crisis besides pregnancyrelated complication. Due to this, there is increase in morbidity and mortality. As there is advancement in the medicine, the mortality rate from SCD is decreased and the lifetime expectancy is increased to 50 years from previous 14 years. ${ }^{4}$ This change in the vital statistics leads to more number of women entering into the reproductive life and becoming pregnant. ${ }^{5}$ Individual studies have shown pregnancies complicated with SCD have multiple maternal and fetal complications, but due to heterogenicity of different studies and pathophysiology of SCD controversies exist regarding the exact effect of SCD on pregnancy and its outcome. Many studies focus primarily on the effect of SCD on maternal and fetal outcomes but patients with sickle cell trait (SCT) also have got increased complications during pregnancy, specifically due to coexisting anemia, dehydration, acidosis, doing vigorous exercise, and living in high altitude. Therefore, we conducted a prospective observational trial to compare the maternal and fetal outcomes among pregnant patients suffering from SCD or SCT.

\begin{abstract}
${ }^{1-4}$ Department of Obstetrics and Gynecology, Veer Surendra Sai Institute of Medical Science and Research, Sambalpur, Odisha, India ${ }^{5}$ Department of Obstetrics and Gynecology, All India Institute of Medical Sciences, Patna, Bihar, India

Corresponding Author:Santosh Kumar Dora, Department of Obstetrics and Gynecology, Veer Surendra Sai Institute of Medical Science and Research, Sambalpur, Odisha, India, Phone: +91 7978279890, e-mail: santoshdora1@gmail.com

How to cite this article: Dora SK, Dandapat AB, Pande $B$, et al. A Prospective Study to Compare the Maternal and Fetal Outcomes among Sickle Cell Disease and Trait Women. J South Asian Feder Obst Gynae 2019;11(6):340-344.

Source of support: Nil

Conflict of interest: None
\end{abstract}

\section{Materials and Methods}

The study was a prospective observational study conducted at VIMSAR, Burla, one of the leading medical colleges of western Odisha. It is a tertiary care hospital with a catchment area of three large states of India. All the pregnant women with documented SCT and SCD delivered during the period from December 2015 to December 2016 were enrolled. Relevant patients' information was entered on a predesigned performa. Patient's information, such as name, age, and address, was entered. Parity, gestational age at delivery, details of obstetrics history, antenatal complications, history of blood transfusion during pregnancy, sickle cell crisis, mode of delivery, and past and medical history were noted. 
All patients were examined in terms of general, systemic, and obstetric examination at the time of admission. According to the standard hospital protocol, all patients were managed. They were promptly treated for any sickle cell crisis or any other complications. Blood transfusion was carried out for hemoglobin less than $6 \mathrm{~g} \%$. Patients were monitored in a high dependency or intensive care unit according to the clinical situation demands. Information on the mode of delivery, indications of the cesarean section, maternal and fetal complications, if any, was noted. Fetal outcomes like birth weight, live born or still born, and babies requiring neonatal intensive care unit (NICU) monitoring were noted. All were grouped into either SCT or SCD according to the high-performance liquid chromatography report. Cases with heterozygous combination were excluded from the study. All maternal and fetal parameter between the two groups (SCD and SCT) were compared.

\section{Statistical Analysis}

For this observational prospective, trial patients who had delivered were grouped into SCD and SCT. All the relevant information was tabulated in a Microsoft Excel sheet. The statistical analysis was carried out in the SPSS 12 version software. The Student's $t$ test, the Chi-square test, and the Fisher's exact test were applied wherever applicable.

\section{Results}

During the study period, 3,780 women were admitted to the labor room or ward and subsequently they had delivered, out of which 178 women (4.7\%) were diagnosed to have sickle cell anemia (SCD and SCT). Baseline characteristics of the mother with SCD and SCT are summarized in Table 1. When these patients were evaluated, 59 (33.14\%) were found to have SCD and 119 (66.85\%) with SCT. Both these groups were comparable in term of age, parity, and number of previous abortions. The mean ages of SCD and SCT patients were $23.4 \pm 3.58$ years and $23.6 \pm 3.81$ years, respectively. Both SCD $(10,16.94 \%)$ and SCT $(14,11.76 \%)$ patients had similar previous history of abortion. When we analyzed these patients, we found that $36(61.01 \%)$ SCD and SCT (38.65\%) patients had hemoglobin value less than $10 \mathrm{~g} \%$. On further analysis of the hemoglobin value, $14(23.7 \%)$ SCD patients had hemoglobin value of less than $8 \mathrm{~g} \%$. The corresponding figure for SCT was $4(3.4 \%)(p=0.000)$. Because of more number of severely anemic patients in the SCD group, significant number of $\operatorname{SCD}(36,61 \%)$ patients required blood transfusion during pregnancy. In the SCT group, 8 (6.7\%) patient had blood transfusion $(p=0.000)$ for anemia. As we know, patients with SCD were more prone to develop sickle cell crisis, which could be chest crisis, painful crisis, and hemolytic crisis. The commonest crisis observed during pregnancy was painful crisis. A total of 17 (28.8\%) of SCD and 5 (4.2\%) of SCT patients presented with painful crisis $(p=0.000)$. Acute chest syndrome was the next common crisis developed in nine (15.3\%) of SCD and one $(0.8 \%)$ of SCT pregnant patients $(p=0.000)$. Hemolytic crisis was seen in four $(6.8 \%)$ of SCD patients and no patient with SCT presented with the same problem $(p=0.010)$. Besides the sickle cell crisis, there was also statistically significant difference in the incidence of hypertension, preeclampsia, and jaundice between the two groups $(p=0.000)$. In the SCD group, the incidences of hypertension, preeclampsia, and jaundice were 11 (18.6\%), 19 (32.2\%), and 15 (25.4\%), respectively. The figures for SCT were 5 (4.2\%) and 7 (5.9\%), respectively. No patient in the SCT group developed jaundice. Though incidence of deep vein thrombosis is rare compared to sickle cell crisis, two (3.4\%)
Table 1: Maternal complications and outcomes

\begin{tabular}{llll}
\hline Characteristics & $S C D(n=59)$ & $S C T(n=119)$ & $p$ value \\
\hline Age & $23.4 \pm 3.58$ & $23.6 \pm 3.81$ & NS \\
Number of abortion & $10(16.94 \%)$ & $14(11.76 \%)$ & NS \\
Hemoglobin level $<8 \mathrm{~g} \%$ & $14(23.7 \%)$ & $4(3.4 \%)$ & 0.000 \\
$8-10 \mathrm{~g} \%$ & $22(37.3 \%)$ & $42(35.3 \%)$ & $\mathrm{NS}$ \\
$>10 \mathrm{~g} \%$ & $23(39 \%)$ & $73(61.3 \%)$ & $\mathrm{NS}$ \\
Blood transfusion & $36(61 \%)$ & $8(6.7 \%)$ & 0.000 \\
Chest crisis & $9(15.3 \%)$ & $1(0.8 \%)$ & 0.000 \\
Painful crisis & $17(28.8 \%)$ & $5(4.2 \%)$ & 0.000 \\
Hemolytic crisis & $4(6.8 \%)$ & $0(0 \%)$ & 0.011 \\
Preeclampsia & $19(32.2 \%)$ & $7(5.9 \%)$ & 0.000 \\
Hypertension & $11(18.6 \%)$ & $5(4.2 \%)$ & 0.000 \\
Deep vein thrombosis & $2(3.4 \%)$ & $0(0 \%)$ & $\mathrm{NS}$ \\
Jaundice & $15(25.4 \%)$ & $0(0 \%)$ & 0.000 \\
Placental abruption & $3(5.1 \%)$ & $3(2.5 \%)$ & $\mathrm{NS}$ \\
Oligohydramnios & $11(18.6 \%)$ & $12(10.1 \%)$ & $\mathrm{NS}$ \\
PPROM & $3(5.1 \%)$ & $4(3.4 \%)$ & $\mathrm{NS}$ \\
Maternal death & $8(13.6 \%)$ & $1(0.8 \%)$ & 0.000 \\
Period of gestation & $36.5 \pm 2.76$ & $38.2 \pm 2.10$ & 0.000 \\
Mode of delivery & & & \\
LSCS & $29(49.2 \%)$ & $50(42 \%)$ & $\mathrm{NS}$ \\
Vaginal & $30(50.8 \%)$ & $69(58 \%)$ & $\mathrm{NS}$ \\
\hline SCD sickleceldisease $S C T, 51 \%$ &
\end{tabular}

$\mathrm{SCD}$, sickle cell disease; $\mathrm{SCT}$, sickle cell trait; NS, not significant; PPROM, preterm premature rupture of membrane

patients in SCD and none in the SCT group had this complication. We didn't find any statistical correlation for this in both the groups $(p=0.109)$. Other obstetrical complications during pregnancy like placental abruption $3(5.1 \%)$ vs $3(2.5 \%)$, preterm premature rupture of membrane $3(5.1 \%)$ vs 4 (3.4\%), and oligohydramnios 11 (18.6\%) vs 12 (10.1\%), seen between SCD and SCT groups, were comparable with no statistical difference between them. In SCD and SCT patients, the mean periods of gestations when they delivered were $36.5 \pm 2.76$ weeks and $38.2 \pm 2.10$ weeks, respectively, with a $p$ value of 0.000 . Among SCD patients, 29 (49.2\%) had cesarean delivery and $30(50.8 \%)$ had vaginal delivery for different maternal indications. The figures for SCT were 50 (42\%) and 69 (58\%), respectively. The most common indication for the cesarean section was fetal distress with oligohydramnios in both the groups. In SCD 17 (28.87\%) and in SCT 29 (24.36\%) patients required the cesarean section for the abovementioned indication. Eight pregnant mothers in the SCD consisting of $8(13.6 \%)$ have died of the disease compared to $1(0.8 \%)$ maternal death is seen among SCT patients. Out of eight deaths in SCD group, four (50\%) died because of acute chest syndrome (ACS) and one patient with SCT also died because of ACS. In the rest four deaths that had occurred in SCD patients, two patients presented with painful crisis, one patient had deep vein thrombosis, and in one patient no apparent cause was identified. The details of maternal complications and outcomes are summarized in Table 1. There is a statistically significant difference between the fetal outcomes (live born vs still born, birth weight, and NICU admission) between the two groups with a $p$ value of 0.000 . Detail of it is presented in Table 2. Ten (16.9\%) of SCD and one $(0.8 \%)$ of SCT patients had intrauterine death. Average birth weight of babies delivered in the SCD group was $2142 \pm 557.45 \mathrm{~g}$ and for SCT it was $2684 \pm 551.23 \mathrm{~g}$. In SCD 40 (67.8\%) and in SCT 30 (25.2\%) babies had low birth weight. As more babies delivered were small for gestation in the SCD group, 
15 (25.4\%) required neonatal intensive care monitoring. The figure for SCT is $14(11.8 \%)$, with a $p$ value of 0.000 .

\section{Discussion}

Sickle cell anemia is a qualitative abnormality of hemoglobin synthesis. According to the management prospective, it can be categorized into SCD and SCT. Patients with SCD are at increased risk for maternal fetal complications as they are more prone to hypoxia, vascular stasis, and microvascular thrombi. Even studies suggest that patients with SCT are also at risk for complications during pregnancy. ${ }^{6}$ As better healthcare facility is available across the globe, more women are becoming pregnant. Earlier studies suggest that patients with sickle cell anemia are at increased risk for spontaneous abortion during pregnancy. ${ }^{7}$ It is hypothesized that placental vasculopathy could be the cause behind increase spontaneous abortion in patients with SCD. In our study, the incidence of spontaneous pregnancy loss between SCD and SCT was almost similar. The patients with SCD when compared to SCT are more prone to develop obstetrical complication during pregnancy apart from complications that will arise because of sickle cell. We have found significantly increased incidence of pregnancy-induced hypertension, 11 (18.6\%) vs 5 (4.2\%), and preeclampsia, $19(32.2 \%)$ vs 7 (5.9\%), among SCD patients compared to SCT patients. Conflicting reports are present in the literature regarding the incidence of preeclampsia in SCD women. Studies conducted by Villers et al., ${ }^{8}$ Chakravarty et al., ${ }^{9}$ and Al Jama et al. ${ }^{10}$ confirmed our findings on preeclampsia. Though others have reported no increased incidence of preeclampsia but this is due to the fact that those studies are not sufficiently powerful to make a firm conclusion in this regard. ${ }^{7,11}$ These patients are more prone to develop placental abruption due to increased incidence of vasculopathy compared to general population. ${ }^{8}$ The incidence of placental abruption in general population was $0.6 \% .{ }^{12}$ In the present study, the incidence of abruption was 3 (5.1\%) in SCD and 3 (2.5\%) in SCT patients, which is four to eight times higher than the general population. Though there is no statistically significant difference between the incidence of placental abruption among the SCD and SCT patients, both groups have significantly higher risk for development of abruption during pregnancy. Due to qualitative defect in hemoglobin, they are more prone for destruction resulting in anemia with increased incidence of blood transfusion and iron overload. No consensus emerged over the role of prophylactic blood transfusion. Royal College of Obstestrics and Gynaecolgy (RCOG) doesn't advice the use of prophylactic blood transfusion during pregnancy. ${ }^{13}$ We have found that $36(61 \%)$ of SCD patients required blood transfusion during pregnancy. Most of the studies conducted on SCD during pregnancy report similarly high incidence of blood transfusion during pregnancy. ${ }^{11}$ Recent meta-analysis by Oteng-Ntim et al..$^{14,15}$ and Malinowski et al. ${ }^{16}$ showed decrease in maternal and fetal mortality and decreased episodes of sickle cell crisis and preterm

Table 2: Fetal outcomes

\begin{tabular}{llll}
\hline Fetal parameter & SCD & SCT & p value \\
\hline IUGR & $33(55.9 \%)$ & $21(17.6 \%)$ & 0.000 \\
Birth weight & $2142 \pm 557.45 \mathrm{~g}$ & $2684 \pm 551.23 \mathrm{~g}$ & 0.000 \\
Low birth weight & $40(67.8 \%)$ & $30(25.2 \%)$ & 0.000 \\
Still born & $10(16.9 \%)$ & $1(0.8 \%)$ & 0.000 \\
NICU admission & $15(25.4 \%)$ & $14(11.8 \%)$ & 0.000 \\
\hline
\end{tabular}

$\mathrm{SCD}$, sickle cell disease; SCT, sickle cell trait; NS, not significant; IUGR, intrauterine growth restriction; NICU, new born intensive care unit delivery in patients who had received prophylactic blood transfusion during pregnancy. Ngo et al. ${ }^{17}$ and Asma et al. ${ }^{18}$ reported $100 \%$ blood transfusion in SCD patients during pregnancy. Others researchers like Daigavane et al., ${ }^{19}$ Resende Cardoso et al., ${ }^{20}$ Al Kahtani, ${ }^{21}$ and Oteng-Ntim et al. ${ }^{14,15}$ reported $46.6 \%, 51.9 \%, 37.2 \%$, and $23.9 \%$ of patients required blood transfusion during pregnancy. We did not transfuse blood prophylactically in our patients. But prophylactic blood transfusion can be considered in patients with twin pregnancy and previous bad obstetrics history, and patients with end organ damage may be benefited by maintaining the hemoglobin level above $10 \mathrm{~g} / \mathrm{dL}$. Blood transfusion has got its own complications like transfusion reaction, alloimmunization, infection, and iron overload. So, consultation with a hematologist along with an obstetrician is important in this regard. Patients with SCD are at higher risk of developing sickle cell crisis, like painful crisis, ACS, and hemolytic crisis during pregnancy. Painful crisis is the most frequent complication of SCD during pregnancy with $27-50 \%$ of women having a painful crisis during pregnancy. ${ }^{10}$ In our study population, 17 (28.8\%) of SCD women had this problem. They were managed by a multidisciplinary team. They were advised to avoid cold environment, dehydration, excessive exercise, and stress to decrease the incidence of painful crisis. Studies done by Oteng-Ntim et al., ${ }^{14,15}$ Daigavane et al., ${ }^{19}$ Resende Cardoso et al., ${ }^{20}$ Al Kahtani, ${ }^{21}$ and Alayed et al. ${ }^{22}$ describe similarly high incidence of painful crisis in SCD patients. The next common complication occurred during pregnancy is ACS seen in $7-20 \%$ of patients. We have 9 (15.3\%) SCD patients presented with ACS. They should promptly be treated as delay in recognition and treatment can lead to death of the women. In the present study, out of eight maternal deaths four (50\%) patient had ACS. Another study conducted by Daigavane et al. ${ }^{19}$ from India reported pulmonary complication in $10 \%$ of their total population. Similar high incidence of pulmonary complications was also reported by Resende Cardoso et al. ${ }^{20}(29.6 \%)$ in their study populations. Due to the structural defect in the hemoglobin, patients with SCD are at risk of hemolytic crisis during pregnancy. We had four (6.8\%) women presented with hemolytic crisis. Incidences of hematological complications were reported by Oteng-Ntim et al. ${ }^{14,15}(27.5 \%)$, Daigavane et al. ${ }^{19}(13.3 \%)$, Resende Cardoso et al. ${ }^{20}(10 \%)$, Al Kahtani et al. ${ }^{21}(13 \%)$, and Alayed et al. ${ }^{22}(9.5 \%)$ in their respective studies. These women most commonly present with jaundice with the deranged liver function test. This has to be differentiated from HELLP syndrome and severe preeclampsia. The incidence of thromboembolism during pregnancy was 1 in 1,500. ${ }^{23}$ The SCD patients have tendency toward coagulation and vascular stasis, which risk them for the development of thromboembolism. ${ }^{24}$ In our series, two (3.4\%) of SCD women developed deep vein thrombosis during pregnancy, which is 34-41 times of baseline risk of embolism in a normal pregnant women. There is a wide variation in the mortality rate among different parts of the world as reported by Al Kahtani et al. ${ }^{21}(2 \%)$, Alayed et al. ${ }^{22}(0.2 \%)$, and Muganyizi et al. ${ }^{25}$ (11.4\%). In the present study, we had maternal mortality rate of $13.6 \%$ among SCD and $0.8 \%$ in SCT women. This wide variation is due to difficulty in availability of proper healthcare facility in developing countries. Pregnant women with SCD with no complications should be delivered in around $38-40$ weeks. ${ }^{13,26}$ In the present study, the mean period of gestation of delivery was significantly lower, $36.5 \pm$ 2.76 weeks, for SCD compared to $38.2 \pm 2.1$ weeks for SCT women. They should be offered vaginal delivery and the cesarean section should be done for the obstetrical condition (Howard et al. ${ }^{27}$ ). But due to increased maternal and fetal complications during pregnancy, there is an increased incidence of the cesarean section rate among 
both the SCD and SCT patients. In the present study, $49.2 \%$ of SCD and $42 \%$ of SCT women had undergone the cesarean section for various obstetrical indications. Such high incidence of the cesarean section was also reported by Asma et al. ${ }^{18}(91.6 \%)$, Alayed et al. ${ }^{22}$ $(36 \%)$, and Boulet et al..$^{28}(43 \%)$. There is no contraindication for spinal anesthesia; rather, it should be preferred over general anesthesia. Maintenance of hydration and blood loss during delivery should be carefully monitored as they are more responsible for the post-partum hemorrhage. There is increased perinatal mortality rate, NICU admission, and low birth weight among babies delivered from SCD compared to SCT women. There is a high incidence of low birth weight in SCD patients as reported by Daigavane et al. ${ }^{19}(53 \%)$ and Al-Farsi et al. ${ }^{29}$ (32.4\%). We had $67.8 \%$ of SCD and $25 \%$ of SCT patients who had babies with low birth weight. Such high incidence is probably due to poor antenatal check-up during pregnancy, low socioeconomic status, and inadequately supervised pregnancy, which is an additive factor for the poor fetal weight gain during pregnancy. The incidence of still birth is 10 (16.9\%) among SCD women compared to $1(0.8 \%)$ in SCT women. Such high still birth rate is uncommon for the developed countries but other studies conducted in India by Daigavane et al. ${ }^{19}$ and Natu et al. ${ }^{30}$ reported high incidence of still birth rate in SCD women. A meta-analysis conducted by Oteng-Ntim ${ }^{14,15}$ over 26,349 women with SCD showed increased rate of IUGR, preeclampsia, maternal mortality, still birth, and preterm delivery. When we reviewed the literature, there is increased perinatal mortality and IUGR among SCD patients than what we have noticed for our patients. ${ }^{9,10}$ Such high rate of fetal complication among SCD is due to increased incidence of chronic hypoxia, sickle cell crisis, vasculopathy, and early delivery. All factors taken together lead to increase in perinatal morbidity and mortality.

\section{Conclusion}

Sickle cell disease women are more prone to develop sickle cell crisis as well as increased obstetrical complications. To prevent complications during pregnancy, preconceptional counseling should be given to all women with sickle cell anemia. They should be counseled regarding the probable complications during pregnancy. All should be advised to keep a low threshold for seeking medical help. They should be managed by a multidisciplinary approach to decrease the morbidity and mortality of both the fetus and mother.

\section{Clinical Significance}

Pregnant women with SCD pose an increased fetomaternal risk compared to SCT patients. They should be managed by a multidisciplinary team.

\section{Compliance with Ethical Standards}

The study is approved by the institutional review committee of our institution. The name of the committee is Veer Surendra Sai Institutional Research and Ethics Committee (VIREC, website: http:// www.vimsar.ac.in, virec@vimsar.ac.in). The trial registry number of the study is 2014/P-I-RP/78. The study was a prospective study where an informed consent was taken from all the participants. For this study, we didn't receive any financial aid from any organization.

\section{Acknowledgments}

We specially thanks Dr Tushar Kanti Meher for the statistical analysis; without him, preparation of this manuscript seems impossible for us.

\section{REFERENCES}

1. Stuart MJ, Nagel RL. Sickle cell disease. Lancet 2004;364:1343-1360. DOI: 10.1016/S0140-6736(04)17192-4.

2. Serjeant GR. The emerging understanding of sickle cell disease. $\mathrm{Br} J$ Haematol 2001;112(1):3-18. DOI: 10.1046/j.1365-2141.2001.02557.x.

3. Piel FB, Patil AP, Howes RE, et al. Global epidemiology of sickle haemoglobin in neonates: a contemporary geostatistical modelbased map and population estimates. Lancet 2013;381(9861):142-151. DOI: 10.1016/S0140-6736(12)61229-X.

4. Claster S, Vichinsky EP. Managing sickle cell disease. BMJ 2003;327:1151-1155. DOI: 10.1136/bmj.327.7424.1151.

5. Serjeant GR, Hambleton I, Thame M. Fecundity and pregnancy outcome in a cohort with sickle cell-haemoglobin C disease followed from birth. BJOG 2005;112(9):1308-1314. DOI: 10.1111/j.14710528.2005.00678.x.

6. Larrabee KD, Monga M. Women with sickle cell trait are at increased risk for preeclampsia. Am J Obstet Gynecol 1997;177(2):425. DOI: 10.1016/S0002-9378(97)70209-6.

7. Serjeant GR, Loy LL, Crowther M, et al. Outcome of pregnancy in homozygous sickle cell disease. Obstet Gynecol 2004;103(6): 1278-1285. DOI: 10.1097/01.AOG.0000127433.23611.54.

8. Villers MS, Jamison MG, De Castro LM, et al. Morbidity associated with sickle cell disease in pregnancy. Am J Obstet Gynecol 2008;199(2):125. e1-125.e5. DOI: 10.1016/j.ajog.2008.04.016.

9. Chakravarty EF, Khanna D, Chung L. Pregnancy outcomes in systemic sclerosis, primary pulmonary hypertension, and sickle cell disease. Obstet Gynecol 2008;111(4):927-934. DOI: 10.1097/01. AOG.0000308710.86880.a6.

10. Al Jama FE, Gasem T, Burshaid S, et al. Pregnancy outcome in patients with homozygous sickle cell disease in a university hospital, Eastern Saudi Arabia. Arch Gynecol Obstet 2009;280(5):793-797. DOI: 10.1007/ s00404-009-1002-7.

11. Zia S, Rafique M. Comparison of pregnancy outcomes in women with sickle cell disease and trait. J Pak Med Assoc 2013;63(6):743-746.

12. Roberts JM, Myatt $L$, Spong $C Y$, et al. Vitamins $C$ and $E$ to prevent complications of pregnancy-associated hypertension. N Engl J Med 2010;362(14):1282. DOI: 10.1056/NEJMoa0908056.

13. Royal College of Obstetricians and Gynecologists. Management of Sickle Cell Disease in Pregnancy. Royal College of Obstetricians and Gynecologists Green-top Guideline No. 61. July; 2011.

14. Oteng-Ntim E, Ayensah B, Knight M, et al. Pregnancy outcome inpatients with sickle cell disease in the UK - a national cohort study comparing sickle cell anemia ( $\mathrm{HbSS}$ ) with $\mathrm{HbSC}$ disease. $\mathrm{Br} J$ Haematol 2015;169(1):129-137. DOI: 10.1111/bjh.13270.

15. Oteng-Ntim E, Meeks D, Seed PT, et al. Adverse maternal and perinatal outcomes in pregnant women with sickle cell disease: systematic review and meta-analysis. Blood 2015;125(21):3316-3325. DOI: 10.1182/blood-2014-11-607317.

16. Malinowski AK, Shehata N, D'Souza R, et al. Prophylactic transfusion for pregnant women with sickle cell disease: a systematic review and meta-analysis. Blood 2015;126(21):2424-2435.

17. Ngo C, Kayem G, Habibi A, et al. Pregnancy in sickle cell disease: maternal and fetal outcomes in a population receiving prophylactic partial exchange transfusions. Eur J ObstetGynecol Reprod Biol 2010;152(2):138-142. DOI: 10.1016/j.ejogrb.2010.05.022.

18. Asma S, Kozanoglu I, Tarım E, et al. Prophylacticred cell exchange may be beneficial in the management of sickle cell disease in pregnancy. Transfusion (Paris) 2014;55(1):36-44. DOI: 10.1111/trf.12780.

19. Daigavane MM, Jena RK, Kar TJ. Perinatal outcome in sickle cell anemia: a prospective study from India. Hemoglobin 2013;37(6): 507-515. DOI: 10.3109/03630269.2013.828301.

20. Resende Cardoso PS, Lopes Pessoa de Aquiar RA, Vianaa MB. Clinical complications in pregnant women with sickle cell disease: prospective study of factors predicting maternal death or near miss. Rev Bras Hematol Hemoter 2014;36(4):256-263. DOI: 10.1016/ j.bjhh.2014.05.007.

21. Al Kahtani MA, AlQahtani M, Alshebaily MM, et al. Morbidity and pregnancy outcomes associated with sickle cell anemia among 
Saudi women. Int J Gynaecol Obstet 2012;119:224-226. DOI: 10.1016/ j.ijgo.2012.07.008.

22. Alayed N, Kezouh A, Oddy L, et al. Sickle cell disease and pregnancy outcomes: population-based study on 8.8 million births. J PerinatMed 2013;17:1-6.

23. Stein $P$, Hull R, Jayali F, et al. Venous thromboembolism in pregnancy: 21-year trends. Am J Med 2004;117(2):121-125. DOI: 10.1016/ j.amjmed.2004.02.021.

24. Sultan AA, Tata LJ, Grainge MJ, et al. The incidence of first venous thromboembolism in and around pregnancy Using Linked Primary and secondary care data: a population based cohort study from England and comparative meta-analysis. PLoS ONE 2013;8(7):e70310. DOI: 10.1371/journal.pone.0070310.

25. Muganyizi PS, Kidanto H. Sickle cell disease in pregnancy: trend and pregnancy outcomes at a tertiary hospital in Tanzania. PLoS ONE 2013;8(2):e56541. DOI: 10.1371/journal.pone.0056541.
26. Boga Can, Baskent HO. Pregnancy and sickle cell disease: a review of the current literature. Crit Rev Oncol Hematol 2016;98:364-374. DOI: 10.1016/j.critrevonc.2015.11.018.

27. Howard J, Oteng-Ntim E. The obstetric management of sickle cell disease. Best Pract Res Clin Obstet Gynaecol 2012;26(1):25-36. DOI: 10.1016/j.bpobgyn.2011.10.001.

28. Boulet SL, Okoroh EM, Azonobi I, et al. Sickle cell disease in pregnancy: maternal complications in a Medicaid-enrolled population. Matern. Child Health J 2013;17(2):200-207. DOI: 10.1007/s10995-012-1216-3.

29. Al-Farsi SH, Al-Khabori MK, Al-Hunieni MN, et al. Fetal outcomes in pregnant women with sickle cell disease. Saudi Med J 2014;35(5): 472-476.

30. Natu N, Khandelwal S, Kumar R, et al. Maternal and perinatal outcome of women with sickle cell disease of a tribal population in Central India. Hemoglobin 2014;38(2):91-94. DOI: 10.3109/03630269.2013. 869501. 